IJMMS 31:12 (2002) 745-750

PII. S0161171202201246

http://ijmms.hindawi.com

(c) Hindawi Publishing Corp.

\title{
ON THE FIRST POWER MEAN OF $L$-FUNCTIONS WITH THE WEIGHT OF GENERAL KLOOSTERMAN SUMS
}

\author{
ZHANG WENPENG
}

Received 20 January 2002

\begin{abstract}
The main purpose of this paper is using the estimates for character sums and the analytic method to study the first power mean of Dirichlet $L$-functions with the weight of general Kloosterman sums, and give an interesting asymptotic formula.
\end{abstract}

2000 Mathematics Subject Classification: 11L05, 11M20.

1. Introduction. Let $q \geq 2$ be an integer, $x$ denotes a Dirichlet character modulo $q$. For any integers $m$ and $n$, we define the general Kloosterman sums $S(m, n, \chi, q)$ as follows:

$$
S(m, n, \chi, q)=\sum_{a=1}^{q} \chi(a) e\left(\frac{m a+n \bar{a}}{q}\right),
$$

where $\bar{a}$ denotes the inverse of $a$ modulo $q$ and $e(y)=e^{2 \pi i y}$. This summation is very important, because it is a generalization of the classical Kloosterman sums. Many authors had studied the properties of $S(m, n, \chi, q)$. For instance, Chowla [1] and Malyšev [3] obtained a sharper upper bound estimation for $S(m, n, \chi, q)$. That is,

$$
|S(m, n, \chi, p)| \ll(m, n, p)^{1 / 2} p^{1 / 2+\epsilon},
$$

where $p$ is a prime, $\epsilon$ is any fixed positive number, and $(m, n, p)$ denotes the greatest common divisor of $m, n$, and $p$. But for an arbitrary composite number $q$, we do not know how large $|S(m, n, \chi, q)|$ is. In fact the value of $|S(m, n, \chi, q)|$ is quite irregular if $q$ is not a prime. The main purpose of this paper is to obtain some good distribution properties of $|S(m, n, \chi, q)|$ in some weight mean value problems. For convenience, in this paper we always suppose $q \geq 3$ be an integer and $L(s, \chi)$ denotes the Dirichlet $L$-function corresponding to character $\chi \bmod q$. Then we can use the estimates for character sums and the analytic method to prove the following main result.

THEOREM 1.1. For any integers $m$ and $n$ with $(m n, q)=1$, we have the asymptotic formula

$$
\sum_{X \neq \chi_{0}}|S(m, n, \chi, q)|^{2} \cdot|L(1, \chi)|=C \cdot \phi^{2}(q)+O\left(q^{3 / 2} \cdot d^{2}(q) \cdot \ln ^{2} q\right),
$$

where

$$
C=\prod_{p}^{\prime}\left[1+\frac{\left(\begin{array}{l}
2 \\
1
\end{array}\right)^{2}}{4^{2} \cdot p^{2}}+\frac{\left(\begin{array}{l}
4 \\
2
\end{array}\right)^{2}}{4^{4} \cdot p^{4}}+\cdots+\frac{\left(\begin{array}{c}
2 n \\
n
\end{array}\right)^{2}}{4^{2 n} \cdot p^{2 n}}+\cdots\right]
$$


is a constant, $\sum_{X \neq \chi_{0}}$ denotes the summation over all nonprincipal characters modulo $q$, $\prod_{p}^{\prime}$ denotes the product over all primes $p$ with $(p, q)=1, d(q)$ is the divisor function, $\phi(q)$ is the Euler function, and $\left(\begin{array}{c}2 n \\ n\end{array}\right)=(2 n) ! /(n !)^{2}$.

For general integer $k \geq 2$, whether there exists an asymptotic formula for

$$
\sum_{\chi \neq \chi_{0}}|S(m, n, \chi, q)|^{2 k}|L(1, \chi)|
$$

is an unsolved problem.

2. Some lemmas. In order to complete the proof of Theorem 1.1, we need the following lemmas.

LEMMA 2.1. For any integer $q \geq 3$, we have the estimate

$$
\sum_{d \mid q} d^{1 / 2} \sum_{s=1}^{q / d-1}\left|\sum_{\chi \neq \chi} \chi(s d+1)\right| L(1, \chi)||=O\left(q \cdot d(q) \cdot \ln ^{2} q\right) .
$$

Proof. Let $N=q^{3 / 2}, \chi$ be a nonprincipal character $\bmod q$ and $A(\chi, y)=$ $\sum_{N<n \leq y} \mathcal{X}(n)$. Then by Abel identity and Pólya-Vinogradov inequality, we have

$$
\begin{aligned}
L(1, \chi) & =\sum_{n \leq N} \frac{\chi(n)}{n}+\int_{N}^{+\infty} \frac{A(\chi, y)}{y^{2}} d y \\
& =\sum_{n \leq N} \frac{\chi(n)}{n}+O\left(\frac{\ln q}{q}\right) .
\end{aligned}
$$

So that

$$
|L(1, \chi)|=\left|\sum_{n \leq N} \frac{\chi(n)}{n}\right|+O\left(\frac{\ln q}{q}\right) .
$$

On the other hand, let $r(n)$ be a multiplicative function defining by

$$
r\left(p^{\alpha}\right)=\frac{\left(\begin{array}{c}
2 \alpha \\
\alpha
\end{array}\right)}{4^{\alpha}}, \quad r(1)=1
$$

where $p$ is a prime and $\alpha$ is any positive integer. For the function $r(n)$, it is easy to prove that

$$
\begin{aligned}
\sum_{d \mid n} r(d) \cdot r\left(\frac{n}{d}\right) & =1, \\
\left(\sum_{n \leq N} \frac{\chi(n) r(n)}{n}\right)^{2} & =\sum_{m \leq N} \sum_{n \leq N} \frac{\chi(n m) r(m) r(n)}{m n} \\
& =\sum_{n \leq N} \frac{\chi(n)}{n}+\sum_{N<n \leq N^{2}} \frac{\chi(n) r(n, N)}{n},
\end{aligned}
$$


where

$$
r(n, N)=\sum_{\substack{d \mid n \\ d, n / d \leq N}} r(d) \cdot r\left(\frac{n}{d}\right)
$$

Note that the triangle inequalities

$$
|a|-|b| \leq|a \pm b|, \quad|a \pm b| \leq|a|+|b|,
$$

from (2.3), (2.6), Cauchy inequality, and the orthogonality relationship for character sums

$$
\begin{aligned}
\sum_{x \bmod q} \chi(n) & = \begin{cases}\phi(q), & \text { if } n \equiv 1 \bmod q ; \\
0, & \text { otherwise }\end{cases} \\
\sum_{a=1}^{q} \chi(a) & = \begin{cases}\phi(q), & \text { if } \chi=\chi_{0} \\
0, & \text { otherwise }\end{cases}
\end{aligned}
$$

we have

$$
\begin{aligned}
& \sum_{d \mid q} d^{1 / 2} \sum_{s=1}^{q / d-1}\left|\sum_{\chi \neq \chi_{0}} \chi(s d+1)\right| L(1, \chi)|| \\
& =\sum_{d \mid q} d^{1 / 2} \sum_{s=1}^{q / d-1}\left|\sum_{\chi \neq \chi_{0}} \chi(s d+1)\right| \sum_{n \leq N} \frac{\chi(n)}{n}|+O(\ln q)| \\
& =\sum_{d \mid q} d^{1 / 2} \sum_{s=1}^{q / d-1}\left|\sum_{X} \chi(s d+1)\right| \sum_{n \leq N} \frac{x(n)}{n}||+O(q \cdot d(q) \cdot \ln q) \\
& \ll \sum_{d \mid q} d^{1 / 2} \sum_{s=1}^{q / d-1}\left|\sum_{X} x(s d+1)\left(\left|\sum_{n \leq N} \frac{x(n)}{n}\right|-\left|\sum_{n \leq N} \frac{\chi(n) r(n)}{n}\right|^{2}\right)\right| \\
& +\left.\sum_{d \mid q} d^{1 / 2} \sum_{s=1}^{q / d-1}\left|\sum_{X} x(s d+1)\right| \sum_{n \leq N} \frac{\chi(n) r(n)}{n}\right|^{2} \mid+q \cdot d(q) \cdot \ln q \\
& \ll \sum_{d \mid q} d^{1 / 2} \cdot \frac{q}{\sqrt{d}} \cdot\left[\sum_{x}\left(\left|\sum_{n \leq N} \frac{x(n)}{n}\right|-\left|\sum_{n \leq N^{2}} \frac{x(n) r(n, N)}{n}\right|\right)^{2}\right]^{1 / 2} \\
& +\phi(q) \sum_{d \mid q} d^{1 / 2} \sum_{s=1}^{q / d-1} \sum_{\substack{m \leq N \\
(s d+1) m \equiv n \bmod q}}^{\prime} \sum_{\substack{n \leq N \\
\prime}} \frac{r(m) r(n)}{m n}+q \cdot d(q) \cdot \ln q \\
& \ll q \cdot d(q) \cdot\left[\sum_{X}\left|\sum_{N<n \leq N^{2}} \frac{\chi(n) r(n, N)}{n}\right|^{2}\right]^{1 / 2}+q \cdot d(q) \cdot \ln q \\
& +\phi(q) \sum_{d \mid q} d^{1 / 2} \sum_{\substack{m \leq N \\
m=n \bmod d \\
m \neq n}}^{\prime} \sum_{\substack{n \leq N \\
m n}}^{r(m) r(n)}
\end{aligned}
$$




$$
\begin{aligned}
& \ll q^{3 / 2} \cdot d(q) \cdot\left(\sum_{\substack{N<m \leq N^{2} \\
m=n \bmod q}}^{\prime} \sum_{\substack{N<N^{2} \\
m n}}^{\prime} \frac{r(m, N) \cdot r(n, N)}{m n}+q \cdot d(q) \cdot \ln ^{2} q\right. \\
& \ll q \cdot d(q) \cdot \ln ^{2} q .
\end{aligned}
$$

This proves Lemma 2.1.

LEMMA 2.2. For any integer $q \geq 3$, we have the asymptotic formula

$$
\sum_{X \neq \chi_{0}}|L(1, \chi)|=C \cdot \phi(q)+O\left(q^{1 / 2} \cdot \ln q\right),
$$

where

$$
C=\sum_{n=1}^{\infty} \frac{r^{2}(n)}{n^{2}}=\prod_{p}^{\prime}\left[1+\frac{\left(\begin{array}{l}
2 \\
1
\end{array}\right)^{2}}{4^{2} \cdot p^{2}}+\frac{\left(\begin{array}{l}
4 \\
2
\end{array}\right)^{2}}{4^{4} \cdot p^{4}}+\cdots+\frac{\left(\begin{array}{c}
2 n \\
n
\end{array}\right)^{2}}{4^{2 n} \cdot p^{2 n}}+\cdots\right]
$$

is an absolute constant.

Proof. Let $N=q^{3 / 2}$. Then from (2.3), (2.6), the orthogonality relationship for character sums, and the method of proving Lemma 2.1, we have

$$
\begin{aligned}
& \sum_{\chi \neq \chi_{0}}|L(1, \chi)|=\sum_{\chi \neq \chi_{0}}\left|\sum_{n \leq N} \frac{\chi(n)}{n}\right|+O(\ln q) \\
& =\sum_{\chi}\left|\sum_{n \leq N} \frac{\chi(n) r(n)}{n}\right|^{2}+O(\ln q) \\
& +\sum_{\chi}\left(\left|\sum_{n \leq N} \frac{\chi(n)}{n}\right|-\left|\sum_{n \leq N} \frac{\chi(n) r(n)}{n}\right|^{2}\right) \\
& =\phi(q) \cdot \sum_{\substack{m \leq N \\
m=n \bmod q}}^{\prime} \sum_{\substack{n \leq N \\
m n}} \frac{r(m) \cdot r(n)}{m n}+O(\ln q) \\
& +O\left(\sum_{X}\left|\sum_{N<n \leq N^{2}} \frac{\chi(n) \cdot r(n, N)}{n}\right|\right) \\
& =\phi(q) \cdot \sum_{\substack{n=1 \\
(n, q)=1}}^{\infty} \frac{r^{2}(n)}{n^{2}}+O\left(\ln ^{2} q\right) \\
& +O\left(q^{1 / 2}\left(\sum_{x}\left|\sum_{N<n \leq N^{2}} \frac{x(n) \cdot r(n, N)}{n}\right|^{2}\right)^{1 / 2}\right) \\
& =\phi(q) \cdot \sum_{\substack{n=1 \\
(n, q)=1}}^{\infty} \frac{r^{2}(n)}{n^{2}}+O\left(q^{1 / 2} \cdot \ln q\right) \\
& =C \cdot \phi(q)+O\left(q^{1 / 2} \cdot \ln q\right) \text {. }
\end{aligned}
$$

This proves Lemma 2.2. 
LEMMA 2.3. Let $m, n$, and $q$ be integers with $q \geq 3$. Then we have the estimates

$$
S(m, n, q)=\sum_{a=1}^{q} e\left(\frac{m a+n \bar{a}}{q}\right) \ll(m, n, q)^{1 / 2} q^{1 / 2} d(q),
$$

where $\sum_{a}^{\prime}$ denotes the summation over all a such that $(a, q)=1$.

Proof. See [2].

3. Proof of Theorem 1.1. In this section, we will complete the proof of Theorem 1.1. First we have

$$
\begin{aligned}
& \sum_{X \neq \chi_{0}}|L(1, \chi)| \cdot|S(m, n, \chi, q)|^{2} \\
& \quad=\sum_{r=1}^{q} \sum_{s=1}^{q} e\left(\frac{(r-s) m+(\bar{r}-\bar{s}) n}{q}\right) \sum_{\chi \neq \chi_{0}} \chi(r \bar{s})|L(1, \chi)| \\
& =\sum_{r=1}^{q} \sum_{s=1}^{q} e\left(\frac{r(1-\bar{s}) m+\bar{r}(1-s) n}{q}\right) \sum_{\chi \neq \chi_{0}} \chi(s)|L(1, \chi)| \\
& =\phi(q) \sum_{\chi \neq \chi_{0}}|L(1, \chi)|+\sum_{s=2}^{q} \sum_{r=1}^{q} e\left(\frac{r(1-\bar{s}) m+\bar{r}(1-s) n}{q}\right) \sum_{\chi \neq \chi_{0}} \chi(s)|L(1, \chi)| .
\end{aligned}
$$

From (3.1) and Lemmas 2.2 and 2.3 we get

$$
\begin{aligned}
\sum_{X \neq \chi_{0}}|L(1, \chi)| \cdot|S(m, n, \chi, q)|^{2} \\
=C \cdot \phi^{2}(q)+O\left(q^{3 / 2} \cdot \ln q\right) \\
\quad+O\left(q^{1 / 2} d(q) \sum_{s=2}^{q}((s-1) m,(\bar{s}-1) n, q)^{1 / 2}\left|\sum_{X \neq \chi_{0}} X(s)\right| L(1, x)||\right) .
\end{aligned}
$$

Note that $(m, q)=(n, q)=(s, q)=1$, and $(1-s, q)=(s \bar{s}-s, q)=(s(\bar{s}-1), q)=(\bar{s}-$ $1, q)$, so we have $(s-1, \bar{s}-1, q)=(s-1, q)$. Thus from (3.2) and Lemma 2.1 we obtain the asymptotic formula

$$
\begin{aligned}
& \sum_{X \neq \chi_{0}}|L(1, \chi)| \cdot|S(m, n, \chi, q)|^{2} \\
&= C \cdot \phi^{2}(q)+O\left(q^{3 / 2} \cdot \ln q\right) \\
&+O\left(q^{1 / 2} d(q) \sum_{s=2}^{q}(s-1, q)^{1 / 2}\left|\sum_{\chi \neq \chi_{0}} \chi(s)\right| L(1, x) \mid\right) \\
&= C \cdot \phi^{2}(q)+O\left(q^{3 / 2} \cdot \ln q\right) \\
&+O\left(q^{1 / 2} d(q) \sum_{d \mid q} \sum_{t=1}^{q / d-1} d^{1 / 2}\left|\sum_{x \neq \chi_{0}} x(t d+1)\right| L(1, x) \mid\right) \\
&= C \cdot \phi^{2}(q)+O\left(q^{3 / 2} \cdot d^{2}(q) \cdot \ln ^{2} q\right) .
\end{aligned}
$$

This completes the proof of the theorem. 
ACKNOWLEDGMENT. This work is supported by the National Natural Science Foundation of China (NSFC) and the Shaanxi Province Natural Science Foundation of China (PNSF).

\section{REFERENCES}

[1] S. Chowla, On Kloosterman's sum, Norske Vid. Selsk. Forh. (Trondheim) 40 (1967), 70-72.

[2] T. Estermann, On Kloosterman's sum, Mathematika 8 (1961), 83-86.

[3] A. V. Malyšev, A generalization of Kloosterman sums and their estimates, Vestnik Leningrad. Univ. 15 (1960), no. 13, 59-75 (Russian).

ZHANG WENPENG: RESEARCH CENTER FOR BASIC SCIENCE, XI'AN JIAOTONG UNIVERSITY, XI'AN, SHAANXI, CHINA

E-mail address: wpzhang@nwu.edu.cn 


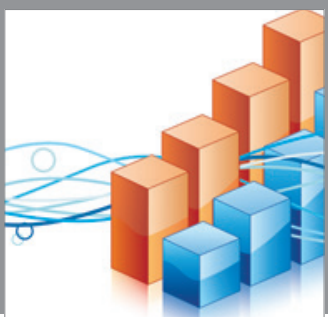

Advances in

Operations Research

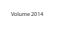

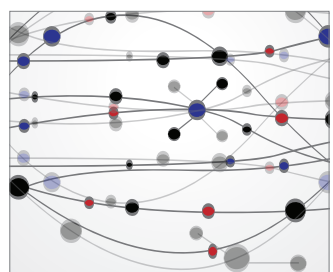

\section{The Scientific} World Journal
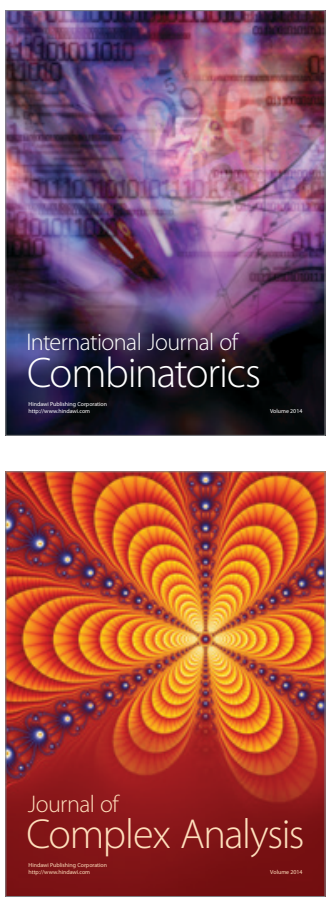

International Journal of

Mathematics and

Mathematical

Sciences
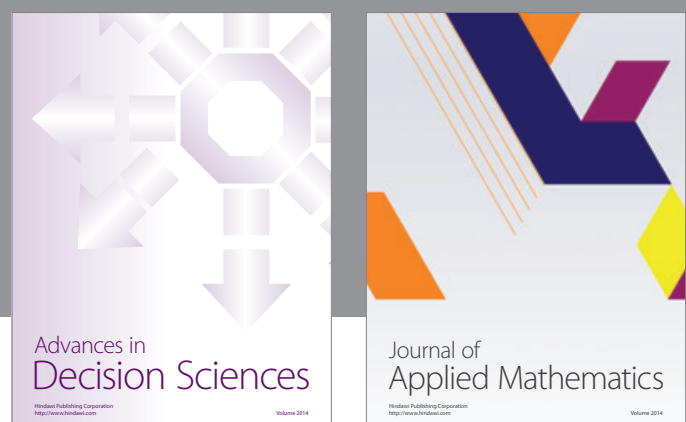

Journal of

Applied Mathematics
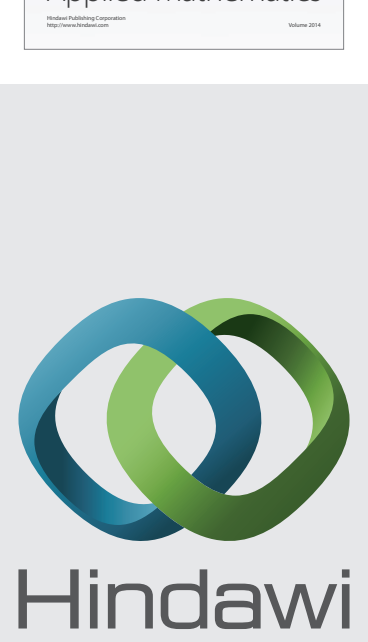

Submit your manuscripts at http://www.hindawi.com
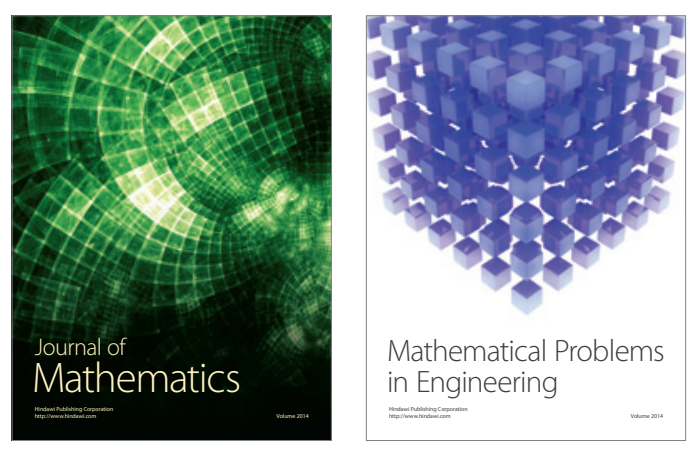

Mathematical Problems in Engineering
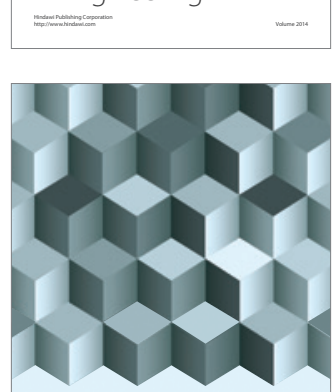

Journal of

Function Spaces
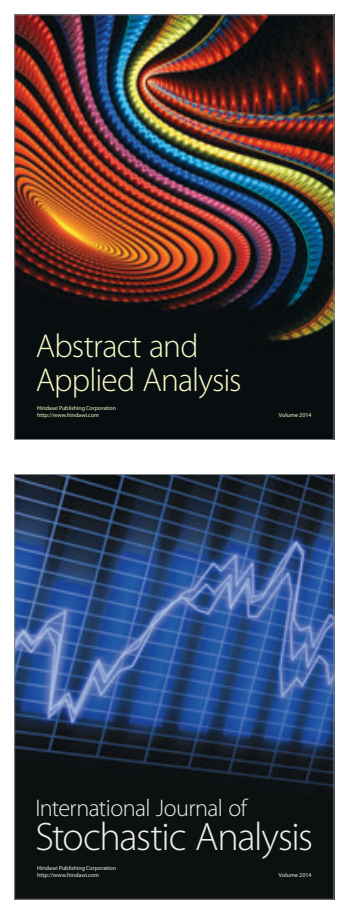

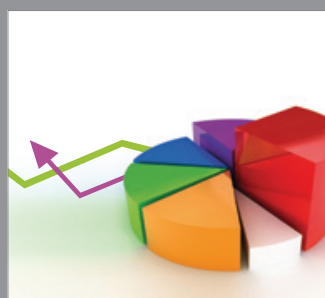

ournal of

Probability and Statistics

Promensencen
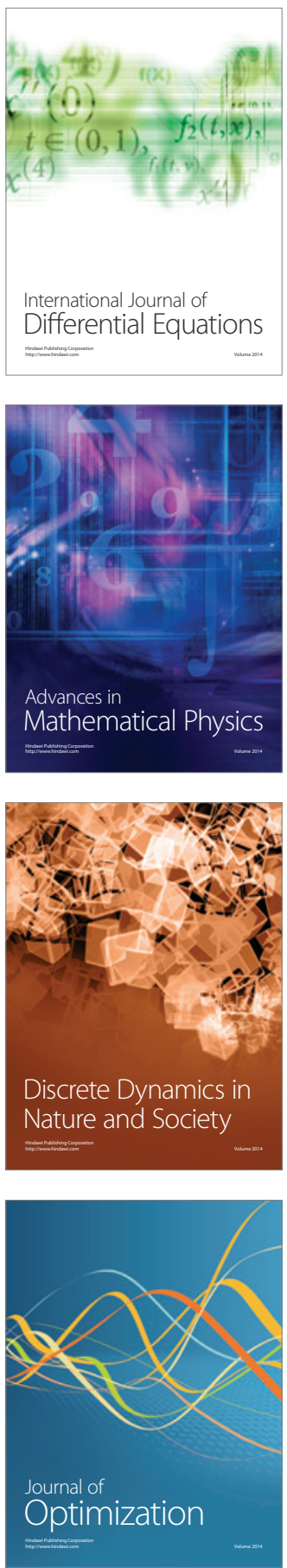